\title{
Effect of a 2 week CPAP treatment on mood states in patients with obstructive sleep apnea: a double-blind trial
}

\author{
Alexander Haensel • Daniel Norman • Loki Natarajan • \\ Wayne A. Bardwell • Sonia Ancoli-Israel • \\ Joel E. Dimsdale
}

Published online: 15 May 2007

(C) Springer-Verlag 2007

\begin{abstract}
Obstructive sleep apnea (OSA) is a common disease with significant medical and psychiatric comorbidities. The literature documenting the effects of continuous positive airway pressure (CPAP) treatment on mood in OSA patients is mixed. We previously observed that 1 week of CPAP treatment did not result in improvements in mood beyond those observed in a group treated with placeboCPAP. This study examined the effect of a 2 week CPAP treatment on mood in a placebo-controlled design in OSA patients. Fifty patients with untreated sleep apnea were evaluated by polysomnography and completed the Profile of Mood States (POMS) pre-/post-treatment. The patients were randomized for 2 weeks to either therapeutic CPAP or placebo-CPAP (at insufficient pressure). Both the thera-
\end{abstract}

\footnotetext{
A. Haensel

Department of General Internal Medicine,

University Hospital Berne,

Bern, Switzerland
}

A. Haensel • W. A. Bardwell • S. Ancoli-Israel • J. E. Dimsdale Department of Psychiatry, University of California,

San Diego, USA

L. Natarajan · W. A. Bardwell · S. Ancoli-Israel · J. E. Dimsdale Moores UCSD Cancer Center,

La Jolla, USA

D. Norman

Pulmonary and Critical Care Medicine, University of California, San Diego, USA

\author{
A. Haensel ( $\square)$ \\ USCD Psychiatry, \\ 9500 Gilman Drive, \\ La Jolla, CA, USA \\ e-mail: ahaensel@ucsd.edu
}

peutic CPAP and the placebo-CPAP groups showed significant improvements in POMS total score, tension, fatigue, and confusion. No significant time $\times$ treatment effect was observed for either group. We could not show a specific beneficial impact of CPAP treatment on mood in OSA patients.

Keywords Obstructive sleep apnea - Mood disorders . Continuous positive airway pressure $\cdot$ POMS

\section{Introduction}

Obstructive sleep apnea (OSA) is a highly prevalent sleep disorder, characterized by repeated disruptions of breathing during sleep. The sleep fragmentation and the accompanying hypoxemia have been linked to many negative consequences including cardiac arrhythmias, nocturnal hypertension, confusion, cognitive impairment, daytime sleepiness, and mood disorders [1]. OSA is now recognized as a common disorder that is associated with major morbidity and mortality [2].

OSA patients regularly show a high prevalence of psychiatric morbidity [3, 4]. Several studies have examined the effect of continuous positive airway pressure (CPAP) on mood in OSA patients, but findings have been inconclusive. Derderian et al. showed in a relatively small group of OSA patients $(n=7)$ a significant drop in the Profile of Mood States (POMS) total mood disturbance after 2 months of CPAP treatment compared with a nonrandomized control group [5]. Similarly, Engleman et al. examined moderate [6] $(n=32)$ and mild [7] $(n=16)$ OSA patients in randomized, placebo-controlled, crossover studies and found an 
improvement in mood after 4 weeks of CPAP treatment. Recently, different research groups have examined the effect of CPAP on the most common mood disorder, depression, in apnea patients. Means et al. showed a significant decrease in the Beck Depression Inventory (BDI) scores 3 months after CPAP treatment [8]. However, the design of this study was not placebo-controlled and it is interesting to note that the improvement in BDI was independent of objectively observed CPAP compliance. Schwartz et al. found that $38 \%$ of a sample of 50 OSA patients were already being treated with antidepressants before CPAP therapy started; these patients had higher BDI scores than the patients without antidepressant medication [9]. Both groups decreased their BDI after 4 to 6 weeks use of CPAP, but the decrease was greater in the patients taking antidepressants. Again, the design was not placebocontrolled and the authors did not evaluate compliance to antidepressant drug treatment.

In contrast to these results, we previously observed no significant difference in change of mood in a placebocontrolled study that compared therapeutic CPAP with placebo-CPAP delivered at insufficient pressure for 1 week [10]. Similar to our results, Borak et al. did not observe any improvement in anxiety, depression, and mental stress in a nonplacebo-controlled study after 3 and 12 months of CPAP and Munoz et al. found no improvement in BDI in 80 OSA patients matched with 80 healthy control subjects after 12 months of CPAP [11, 12]. In summary, as recently noted by Gay et al., results from placebo-controlled studies are inconclusive with regard to the impact of CPAP on $\operatorname{mood}[13]$.

In our previous study design, we wondered if 1 week of treatment was insufficient to improve mood. In this study, we attempted to replicate and extend our prior findings by using a placebo-controlled study design for 2 weeks of treatment. When this study was designed, we had reservations about offering placebo-CPAP for an extensive period of time to patients with moderate to severe sleep apnea; thus we limited this study to a 2-week treatment trial.

\section{Materials and methods}

\section{Patients}

Fifty patients with a history of snoring and daytime sleepiness suggestive of sleep apnea were recruited by advertisement, word of mouth referral, referral from local medical practitioners in the San Diego area, and referral from previous participants to participate in a study evaluating sympathetic nervous system physiology, cognitive dysfunction, and mood in obstructive sleep apnea. We limited enrollment to subjects in the range of 30 to 65 years and with weight $100-200 \%$ of the ideal body weight per Metropolitan Life Insurance tables [14]. Participants $>200 \%$ of the ideal body weight were excluded because of the possibility of confounding by other conditions associated with obesity. Because of the prominent focus on sympathetic nervous system physiology, the sample is somewhat limited in terms of severe obesity, which has attendant effects on sympathetic nervous system physiology and other medical comorbidities such as diabetes, which could confound an analysis of CPAP effects on mood. Potential participants were also excluded if they had a history of heart, liver or renal disease, diabetes, psychosis, narcolepsy, current alcohol or drug abuse, severe asthma or cerebrovascular disease. Patients with a history of depression were excluded. Patients who were taking antihypertensive medication $(n=7)$ had their medication tapered slowly in $2-3$ steps for 3 weeks before admission to enable more meaningful studies of sympathetic nervous system physiology. Those whose blood pressure at baseline exceeded 170/ $105 \mathrm{mmHg}$ off treatment were returned to antihypertensive medication and not studied. The protocol was approved by the University of California, San Diego, Human Subjects Committee. After complete description of the study to the subjects, written informed consent was obtained.

\section{Polysomnography}

Participants had their sleep monitored for an entire night in the General Clinical Research Center (GCRC) Gillin Laboratory of Sleep and Chronobiology (LSC) with polysomnography measuring central and occipital EEG, bilateral electrooculogram, submental and bilateral tibialis electromyogram, naso-oral airflow thermistor and nasal airflow pressure transducer, and thoracic and abdominal excursions with Respitrace respiratory inductive plethysmography. Oxygen saturation was monitored with a pulse oximeter (Biox 3740, Datex-Ohmeda, Louisville, CO) and was analyzed using a computer software (Profox Associates, Escondido, CA).

Sleep recordings were scored according to the criteria of Rechtschaffen and Kales [15]. Apneas were defined as decrements in airflow of $\geqq 90 \%$ from baseline for $\geqq 10 \mathrm{~s}$. Hypopneas were defined as decrements in airflow of $\geqq 50 \%$ but $<90 \%$ from baseline for $\geqq 10 \mathrm{~s}$ [16]. The majority of subjects had solely obstructive type events with only a few subjects showing evidence of central apneas. Those few who had predominantly central apneas $(>50 \%$ of total apneas) were excluded. The number of apneas and hypopneas per hour were calculated to obtain the apnea hypopnea index (AHI). Obstructive sleep apnea was defined an $\mathrm{AHI} \geqq 15$. 
Table 1 Patient characteristics before treatment

\begin{tabular}{lll}
\hline & $\begin{array}{l}\text { CPAP treatment group } \\
(n=25)\end{array}$ & $\begin{array}{l}\text { Placebo-CPAP group } \\
(n=25)\end{array}$ \\
\hline $\begin{array}{l}\text { Age }( \pm \text { SD }) \\
\text { Sex }\end{array}$ & $48.2 \pm 10.2$ & $49.0 \pm 10.6$ \\
$\quad$ Male & 20 & 20 \\
Female & 5 & 5 \\
Race/ethnicity & & \\
White & 14 & 16 \\
Black & 5 & 1 \\
Hispanic & 3 & 3 \\
Asian & 2 & 1 \\
Other & 1 & 4 \\
BMI $( \pm$ SD $)$ & $33.1 \pm 8.2$ & $33.7 \pm 7.5$ \\
AHI $( \pm$ SD $)$ & $63.6 \pm 29.1$ & $58.4 \pm 30.4$ \\
Mean oxygen & $92.9 \pm 4.4$ & $92.8 \pm 4.15$ \\
saturation & & \\
$( \pm$ SD $)$ & & \\
\hline
\end{tabular}

There was no significant difference between the two groups in any of these variables.

Psychological assessment

Participants completed the POMS before randomization and after 2 weeks of either CPAP or placebo treatment. The POMS is a well-established, factor-analytically derived measure of psychological distress for which high levels of reliability and validity have been documented [17]. The POMS consists of 65 adjectives rated on a 5-point scale $(0=$ not at all to $4=$ extremely) that can be consolidated into 6 subscales. Patients are questioned: How often have you been feeling ...during the past week including today? Thus, stating 'extremely' means high fatigue-inertia, depression-dejection, tension-anxiety, anger-hostility, confusion-bewilderment; vigor-activity is scored in reversei.e., high scores indicate beneficial symptoms as opposed to troubling symptoms. The total score adds the first five subscales and subtracts the last subscale.

In addition, a total mood score is calculated by summing scores for the first five subscales and subtracting the score for vigor-activity. The POMS has been used in a variety of chronically ill and well populations, [17-24] including obstructive sleep apnea patients [10, 25-27].
Treatment groups

Patients with OSA were randomized double-blind into two different treatment groups: therapeutic CPAP and placeboCPAP. On the second night of GCRC admission, the patients randomized to the therapeutic CPAP treatment group underwent a standard CPAP titration study. CPAP was started at a pressure of $4 \mathrm{~cm} \mathrm{H}_{2} \mathrm{O}$ and was increased by 1-2 $\mathrm{cm} \mathrm{H}_{2} \mathrm{O}$ increments based on the pressure of apnea, hypopnea, snoring or respiratory effort related arousals. The titration was ended and considered successful when all significant respiratory events were abolished while the patient was supine after the second or third rapid eye movement sleep period, and the patient had spent at least $15 \mathrm{~min}$ of sleep in the final CPAP level. Patients randomized to the placebo-CPAP group underwent a mock titration night, using room air, via modified CPAP equipment that delivered $<1 \mathrm{~cm} \mathrm{H}_{2} \mathrm{O}$ pressure. The equipment used in both groups appeared identical, consisting of a CPAP unit (Aria LX CPAP System, Respironics, Murrysville, PA) and a heated humidifier (Fisher and Pykel HC100, Aukland New Zealand). In the placebo-CPAP group, the CPAP unit was modified as follows: the CPAP machine was set at $3 \mathrm{~cm}$ $\mathrm{H}_{2} \mathrm{O}$ pressure; however, a $3 \mathrm{~mm}$-diameter pressure restrictor was placed downstream in the CPAP tubing and a special nasal mask with 10 one-quarter-inch drilled holes was used to allow for adequate air exchange with the environment. Mask pressure was $0.5 \mathrm{~cm} \mathrm{H}_{2} \mathrm{O}$ at end-expiration and $0 \mathrm{~cm}$ $\mathrm{H}_{2} \mathrm{O}$ on inspiration. The noise level of both treatment conditions was not perceptibly different. After the "titration" night, the patients were sent home with their assigned treatment equipment. Subsequent telephone calls and home visits ensured proper equipment setup and use. All of the home CPAP units had a hidden compliance clock, which determined the amount of time the equipment was used at the designated pressure. The compliance data were downloaded at the end of the treatment period to determine average hours of use per night. Upon completion of the 2-week treatment period, the patients returned to the GCRC-LSC for a final attended polysomnogram, performed with the patients on their assigned therapy. When the study was initially designed in 2000, we had ethical

Table 2 Mean and standard deviations of respiratory variables, sleep time, and sleep efficiency before and after 2 weeks of CPAP treatment

\begin{tabular}{|c|c|c|c|c|c|c|}
\hline & \multicolumn{2}{|c|}{ CPAP treatment group $(n=25)$} & \multicolumn{4}{|c|}{ Placebo-CPAP treatment group $(n=25)$} \\
\hline & >Day 1 & Day 14 & Day 1 & Day 14 & Time & Time $\times$ treatment group \\
\hline AHI & $65.9 \pm 28.6$ & $3.5 \pm 3.4$ & $57.5 \pm 32.1$ & $53.4 \pm 32.9$ & $<0.001$ & $<0.001$ \\
\hline$\% \mathrm{O}_{2}<90$ & $4.3 \pm 7.5$ & $0.00 \pm 0$ & $2.9 \pm 5.2$ & $2.4 \pm 3.5$ & 0.017 & 0.061 \\
\hline Mean $\mathrm{O}_{2}$ saturation & $93.2 \pm 3.9$ & $92.9 \pm 15.8$ & $92.9 \pm 4.4$ & $91.8 \pm 3.9$ & 0.733 & 0.819 \\
\hline Total sleep time (min) & $346 \pm 9$ & $366 \pm 6$ & $340 \pm 8$ & $352 \pm 8$ & 0.019 & 0.704 \\
\hline Sleep efficiency $(\%)$ & $80.3 \pm 2.3$ & $87.5 \pm 1.5$ & $82 \pm 1.6$ & $85.9 \pm 1.6$ & 0.002 & 0.180 \\
\hline
\end{tabular}


Table 3 Pre-/post-treatment POMS scores for therapeutic CPAP and placebo-CPAP groups (mean \pm standard error)

\begin{tabular}{|c|c|c|c|c|c|c|}
\hline \multirow[t]{2}{*}{ POMS scores } & \multicolumn{2}{|c|}{ Therapeutic CPAP group $(n=25)$} & \multicolumn{4}{|c|}{ Placebo-CPAP group $(n=25)$} \\
\hline & Day 1 & Day 14 & Day 1 & Day 14 & Time & Time $\times$ treatment group \\
\hline Tension & $8.0 \pm 1.3$ & $5.7 \pm 1.2$ & $8.1 \pm 1.2$ & $5.2 \pm 0.9$ & 0.012 & 0.967 \\
\hline Depression & $7.2 \pm 2.1$ & $5.4 \pm 2.1$ & $7.0 \pm 1.6$ & $6.2 \pm 2.3$ & 0.226 & 0.287 \\
\hline Fatigue & $9.9 \pm 1.7$ & $5.4 \pm 1.2$ & $10.4 \pm 1.5$ & $7.5 \pm 2.1$ & 0.004 & 0.211 \\
\hline Confusion & $6.9 \pm 0.9$ & $4.3 \pm 0.7$ & $7.0 \pm 0.8$ & $5.5 \pm 1.1$ & 0.008 & 0.150 \\
\hline Vigor & $14.5 \pm 1.3$ & $16.2 \pm 2.1$ & $13.6 \pm 2.1$ & $15.0 \pm 2.1$ & 0.275 & 0.340 \\
\hline Anger & $5.1 \pm 0.9$ & $3.4 \pm 1.0$ & $6.9 \pm 1.4$ & $5.5 \pm 1.2$ & 0.256 & 0.291 \\
\hline Total & $22.6 \pm 6.2$ & $7.9 \pm 6.5$ & $25.8 \pm 7.3$ & $14.8 \pm 8.0$ & 0.013 & 0.141 \\
\hline
\end{tabular}

reservations about imposing a placebo treatment longer than 2 weeks. Instead, we reasoned that slow advance of the duration of placebo treatment would be necessary before one would be comfortable with a month-long placebo treatment, even if that month-long treatment would be the most compelling test of the hypothesis.

\section{Statistical analysis}

Pre-/post-treatment data were analyzed with repeated measures analysis of variance using the SPSS 12.0 software (SPPS, Chicago, IL). Significance was set at $\alpha=0.05$.

\section{Results}

Demographic characteristics

Characteristics of patients are presented in Table 1. The therapeutic CPAP and placebo-CPAP groups did not differ significantly in age, sex, race/ethnicity or BMI. Mean age overall was $48.6 \pm 10.3$ years, mean BMI was $33.4 \pm 7.8$. Of the participants in both treatment groups, $80 \%$ were male. Average nighttime CPAP use was $6.6 \pm 1.2 \mathrm{~h}$ for the therapeutic CPAP group compared to $6.0 \pm 1.3 \mathrm{~h}$ in the placebo-CPAP group. This difference was also not statistically significant.

Sleep apnea characteristics

After 14 days of treatment, $\mathrm{AHI}$ and $\% \mathrm{SaO}_{2}$ decreased significantly in the therapeutic CPAP group, whereas the placebo group did not show any significant improvement (see Table 2).

Mood

Table 3 and Fig. 1 show pre-/post-treatment POMS scores for both groups. There was no significant time $\times$ treatment interaction for any of the POMS subscales. However, a significant time effect was observed for POMS total score, tension, fatigue, and confusion.

Fig. 1 POMS scores in the therapeutic and placebo-CPAP groups at baseline and day 14

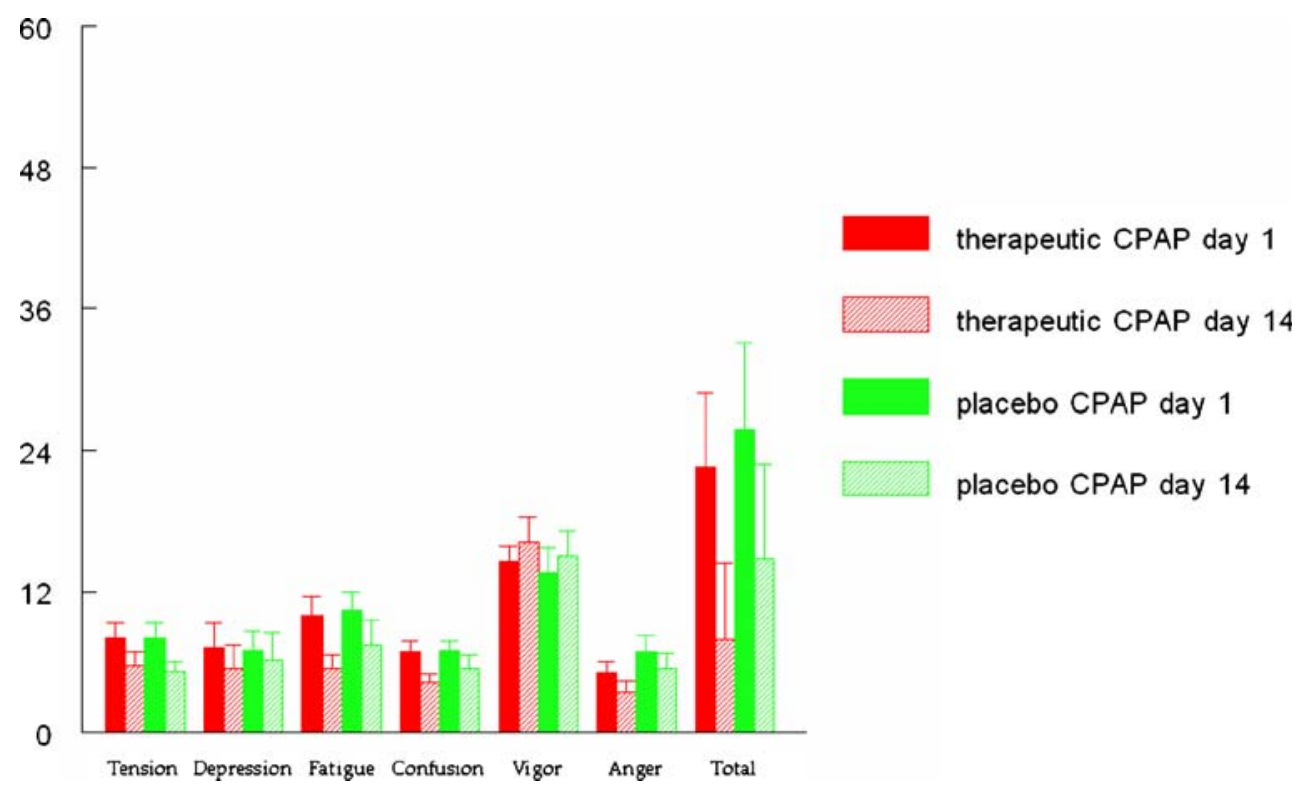




\section{Discussion}

In our sample, only the group that received therapeutic CPAP showed a significant impact of treatment on RDI. However, patients on both therapeutic CPAP and placeboCPAP showed significant improvement on four of the seven POMS mood ratings; there were no time $\times$ treatment interactions. These data suggest a nonspecific effect of CPAP treatment on mood, i.e., the beneficial effects of CPAP on mood may be the result of a placebo effect. This result replicates and extends our previous findings in a different sample of OSA patients; the previous study found that 1 week of CPAP treatment did not have a specific effect on $\operatorname{mood}[10]$.

This result should not be reviewed as a criticism of CPAP. CPAP has clearly been shown to be an effective treatment of sleep apnea [6]. However, our data suggest that an intervention consisting of a caring physician and believable but not functioning CPAP has an enormous beneficial effect on patient mood symptoms - an effect that is independent of CPAP per se. It might be possible that a longer trial of CPAP would demonstrate a beneficial effect on mood.

Our study had several limitations. The POMS was developed initially as a method for assessing changes in psychiatric outpatients; however, it has been widely used in various nonpsychiatric populations. Our sample showed relatively low distress values on most POMS subscales at baseline [17]. This floor effect on total POMS score and subscores could have contributed to our negative finding. The literature reports that in healthy subjects, there is a standard deviation of approximately nine in the depression subscale. Such a large standard deviation in normal values for this score make our observed drop of 1.8 points and 0.8 points on CPAP and placebo, respectively, look rather small in comparison. Another potential limitation in our study is the differential distribution of ethnicity in the treatment groups. We had five African Americans on CPAP and only one on placebo. It is possible that ethnicity affects response differently to therapy; unfortunately, our small numbers do not allow meaningful subgroup analysis.

However as expected, our sample reported higher fatigue and lower vigor scores than in the normative sample. Nonetheless, even in those subscores where our patients were highly symptomatic, we failed to observe a specific effect of CPAP on these symptoms. Is this a function of sample size? While a larger sample size would be desirable, note the powerful effects of the placebo treatment. Table 3 and Fig. 1 demonstrate improvement in all POMS scores in both the active and the placebo treatment groups, although the degree of that improvement was significant only for tension, fatigue, confusion, and total scores. Our interaction analysis suggested no significant difference in the degree of this improvement in the active vs placebo groups. Perhaps with a larger sample we would have been able to perceive subtler specific effects of CPAP on mood, but the tentative conclusion from this study would suggest that the "improvement" in mood symptoms may be a regression to the mean, as has been observed in previous studies of mood [28]. Another limitation could rise from the neurobiological basis of mood change. Response to antidepressant medication typically requires 3 weeks, i.e., underlying changes in the brain in response to antidepressant treatment take time to develop and mature [29]. Perhaps our treatment period of 14 days was too short, i.e., possibly a specific effect of treatment would be evident with a longer treatment.

One might also criticize our exclusion of patients with major medical illnesses other than OSA and hypertension. These criteria resulted in a population that may not be representative of OSA patients normally seen in a sleep clinic. Our population might thus not be considered as "typical" OSA patients because our exclusion criteria served to limit the impact of several potential cofounders that could affect mood. Regardless, our patients suffered from significant sleep apnea verified by AHI (i.e., AHI>50) and significant obesity (BMI $>33)$.

Despite these limitations, this study is one of only a few to examine CPAP effects on mood using a double-blind placebo-controlled randomized trial. We found equal improvements in mood after 2 weeks of therapeutic CPAP or placebo-CPAP treatment, suggesting that any improvements in mood are a result of a nonspecific treatment effect.

Acknowledgments This research was supported by NIH grants AG08415, HL44915, HL36005, and RR00827.

\section{References}

1. Cheshire K, Engleman H, Deary I et al (1992) Factors impairing daytime performance in patients with sleep apnea/hypopnea syndrome. Arch Intern Med 152:538-541

2. Newman AB, Nieto FJ, Guidry U et al (2001) Relation of sleepdisordered breathing to cardiovascular disease risk factors: the Sleep Heart Health Study. Am J Epidemiol 154:50-59

3. Engleman HM, Cheshire KE, Deary IJ et al (1993) Daytime sleepiness, cognitive performance and mood after continuous positive airway pressure for the sleep apnoea/hypopnoea syndrome. Thorax 48:911-914

4. Douglas NJ (1998) Systematic review of the efficacy of nasal CPAP. Thorax 53:414-415

5. Derderian SS, Bridenbaugh RH, Rajagopal KR (1998) Neuropsychologic symptoms in obstructive sleep apnea improve after treatment with nasal continuous positive airway pressure. Chest 94:1023-1027

6. Engleman HM, Martin SE, Deary IJ et al (1994) Effect of continuous positive airway pressure treatment on daytime function in sleep apnoea/hypopnoea syndrome. Lancet 343:572-575 
7. Engleman HM, Martin SE, Deary IJ et al (1997) Effect of CPAP therapy on daytime function in patients with mild sleep apnoea/ hypopnoea syndrome. Thorax 52:114-119

8. Means MK, Lichstein KL, Edinger JD et al (2003) Changes in depressive symptoms after continuous positive airway pressure treatment for obstructive sleep apnea. Sleep Breath 7:31-42

9. Schwartz DJ, Kohler WC, Karatinos G (2005) Symptoms of depression in individuals with obstructive sleep apnea may be amenable to treatment with continuous positive airway pressure. Chest 128:1304-1309

10. Yu BH, Ancoli-Israel S, Dimsdale JE (1999) Effect of CPAP treatment on mood states in patients with sleep apnea. J Psychiatr Res 33:427-432

11. Borak J, Cieslicki JK, Koziej M et al (1996) Effects of CPAP treatment on psychological status in patients with severe obstructive sleep apnoea. J Sleep Res 5:123-127

12. Munoz A, Mayoralas LR, Barbe F, Pericas J, Agusti AG (2000) Long-term effects of CPAP on daytime functioning in patients with sleep apnoea syndrome. Eur Respir J 15:676-681

13. Gay P, Weaver T, Loube D, Iber C, Positive Airway Pressure Task Force, Standards of Practice Committee, American Academy of Sleep Medicine (2006) Evaluation of positive airway pressure treatment for sleep related breathing disorders in adults. Sleep 29:381-401

14. Anonymous (1983) 1983 Metropolitan height and weight tables. Stat Bull Metrop Life Found 64:3-9

15. Rechtshaffen A, Kales A (1968) A manual of standardized terminology: techniques and scoring system for sleep stages of human subjects. UCLA Brain Information Service/Brain Research Institute, Los Angeles

16. Gould GA, Whyte KF, Rhind GB et al (1998) The sleep hypopnea syndrome. Am Rev Respir Dis 137:895-898

17. McNair DM, Lorr M, Droppleman LF (1992) POMS manual: profile of mood states. Educational and Industrial Testing Service, San Diego, CA
18. Cella DF, Tross S, Orav EJ et al (1989) Mood states of patients after the diagnosis of cancer. J Psychosoc Oncol 7:45-54

19. DiLorenzo TA, Bovberg DH, Montgomery GH et al (1999) The application of a shortened version of the profile of mood states in a sample of breast cancer chemotherapy patients. Br J Health Psychol 4:315-325

20. Holland JC, Korzun AH, Tross S et al (1986) Comparative psychological disturbance in patients with pancreatic and gastric cancer. Am J Psychiatry 143:982-986

21. Shumaker SA, Anderson RT, Czajkowski SM (1990) Psychological tests and scales. In: Spilker B (ed) Quality of life assessments in clinical trials. Raven, New York, pp 95-114

22. Spiegel D, Bloom JR, Yalom I (1981) Group support for patients with metastatic cancer: a randomized prospective outcome study. Arch Gen Psychiatry 38:527-533

23. Taylor SE, Lichtmain RR, Wood JV (1984) Attributions, beliefs about control and adjustment to breast cancer. J Pers Soc Psychol 46:486-501

24. Taylor SE, Lichtmain RR, Wood J et al (1985) Illness and treatment-related factors in psychological adjustment to breast cancer. Cancer 55:2503-2513

25. Dickel MJ, Mosko SS (1990) Morbidity cut-offs for sleep apnea and periodic leg movements in predicting subjective complaints in seniors. Sleep 13:155-166

26. Mosko S, Zetin M, Glen S et al (1989) Self-reported depressive symptomatology, mood ratings, and treatment outcome in sleep disorders patients. J Clin Psychol 45:51-60

27. Bardwell WA, Moore P, Ancoli-Israel S et al (2003) Fatigue in obstructive sleep apnea: driven by depressive symptoms instead of apnea severity? Am J Psychiatry 160:350-355

28. Salinsky MC, Storzbach D, Dodrill CB et al (2001) Test-retest bias, reliability, and regression equations for neuropsychological measures repeated over a $12-16$-week period. J Int Neuropsycho Soc 7:597-605

29. Castrén E (2005) Is mood chemistry Nat Rev Neurosci 6:241-6 